\title{
The Role of the Hippo Pathway in Breast Cancer Carcinogenesis, Prognosis, and Treatment: A Systematic Review
}

\author{
Anastasios Kyriazoglou Michalis Liontos Roubini Zakopoulou \\ Maria Kaparelou Anna Tsiara Alkistis Maria Papatheodoridi \\ Rebecca Georgakopoulou Flora Zagouri \\ Department of Clinical Therapeutics, General Hospital Alexandra, Athens, Greece
}

\section{Keywords}

Breast cancer · Hippo pathway · Prognosis · Treatment .

Systematic review

\begin{abstract}
Background: The Hippo pathway is a developmental pathway recently discovered in Drosophila melanogaster; in mammals it normally controls organ development and wound healing. Hippo signaling is deregulated in breast cancer (BC). MST1/2 and LATS1/2 kinases are the upstream molecular elements of Hippo signaling which phosphorylate and regulate the two effectors of Hippo signaling, YAP1 and TAZ cotranscriptional activators. The two molecular effectors of the Hippo pathway facilitate their activity through TEAD transcription factors. Several molecular pathways with known oncogenic functions cross-talk with the Hippo pathway. Methods: A systematic review studying the correlation of the Hippo pathway with BC tumorigenesis, prognosis, and treatment was performed. Results: Recent literature highlights the critical role of Hippo signaling in a wide spectrum of biological mechanisms in BC. Discussion: The Hippo path-
\end{abstract}

way has a crucial position in BC molecular biology, cellular behavior, and response to treatment. Targeting the Hippo pathway could potentially improve the prognosis and outcome of $B C$ patients.

(c) 2020 S. Karger AG, Basel

\section{Introduction}

The Hippo pathway is a developmental pathway discovered recently in Drosophila melanogaster. In mammals' development the Hippo pathway controls organ size, tissue regeneration, wound healing, and maintenance of tissue-specific stem cells [1,2]. In human breast cells, the Hippo pathway has been linked with estrogen receptor alpha to regulate breast cell fate [3]. The molecular elements of the Hippo pathway include MST1/2 and LATS1/2, two kinases which phosphorylate the YAP1 and TAZ cotranscription factors. SAV1 is a mediator which facilitates the proximity of MST1/2 and LATS1/2, thus promoting phosphorylation. MOB1A and MOB1B are additional adapter molecules that promote LATS1/2 


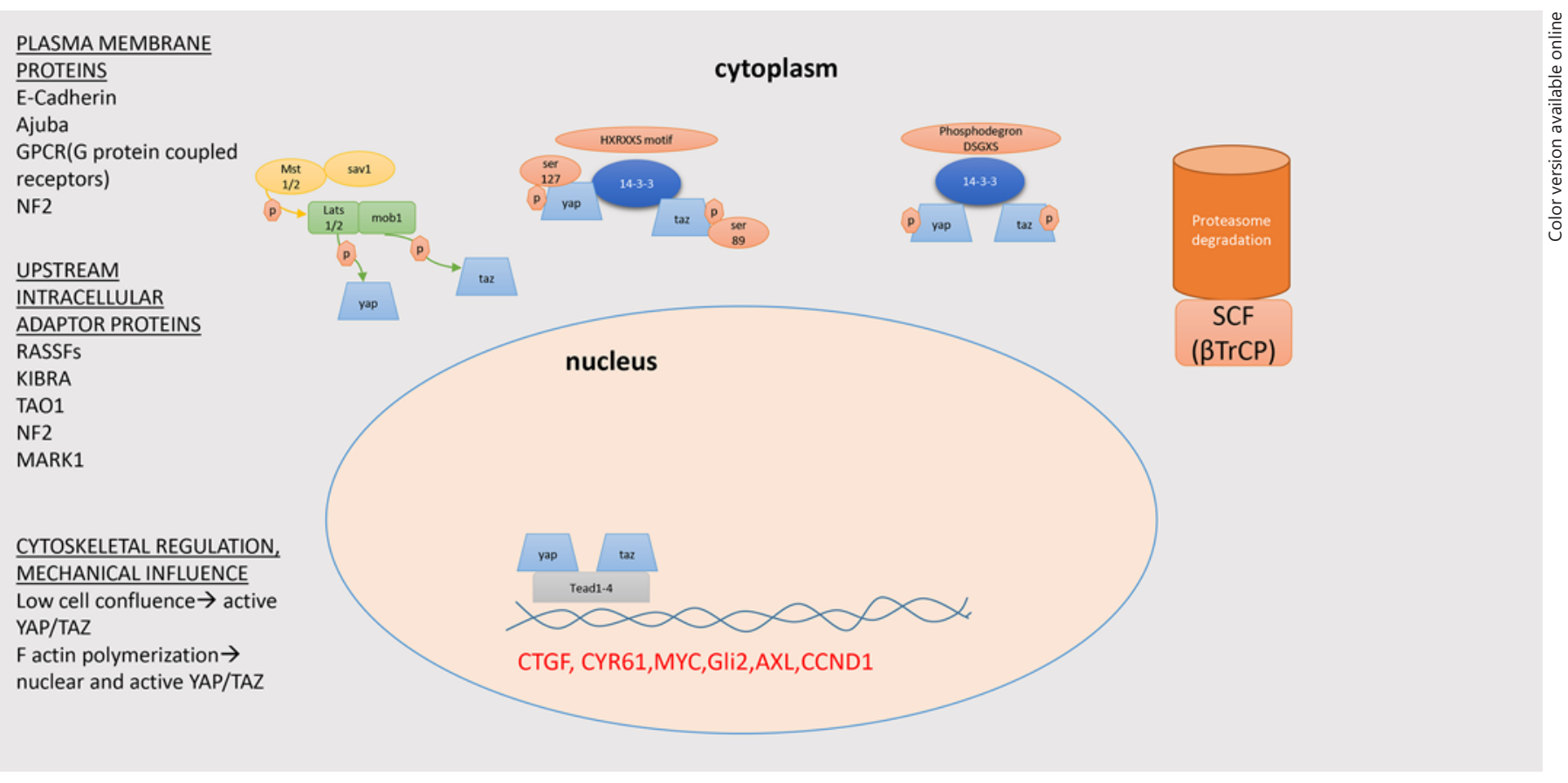

Fig. 1. Schematic presentation of the Hippo pathway and its molecular elements in the cell.

kinase activity. The active hippo kinase cascade results in LATS1/2-mediated phosphorylation at S127 and S381 for YAP1 and S89 and S311 for TAZ. The phosphorylated forms of YAP1 and TAZ cause cytoplasmic retention via their binding to 14-3-3. Ultimately, pYAP and pTAZ undergo proteasomal degradation via the function of SCF complex. In contrast, the unphosphorylated forms of YAP1 and TAZ remain in the nuclei and regulate their function through TEAD transcription factors $[4,5]$.

The Hippo pathway is regulated through several mechanisms (Fig. 1). Plasma membrane proteins like G protein-coupled receptor ligands have been identified as regulators of Hippo signaling [6], with GPER mediating estrogen effects in breast cancer (BC) development [7]. Upstream intracellular adaptor proteins like NF2, RASSFs, and KIBRA interact with MST1/2 and LATS1/2, altering their function and subsequently YAP1 and TAZ localization [8-10]. Mechanical influences cause cytoskeletal remodeling, and YAP1 and TAZ emerge as important factors linking extracellular matrix signals to transcriptional outputs that regulate cell behavior [11, 12]. Furthermore, cross-talk of the Hippo pathway with other pathways such as Wnt/ $\beta$-catenin, TGF- $\beta$, PI3K/ AKT, MAPK, and Jak/Stat is well documented [13-19]. Activation of the Wnt/ $\beta$-catenin pathway leads to Hippo pathway activation via dissociation of YAP1 and TAZ from proteasomal degradation complex $[15,20]$. The Hippo pathway is linked with the JNK pathway (Janus kinase). AP1 (dimmer of JUN and FOS proteins) co-occupies with YAP1 and TAZ in TEAD transcription fac- tors [16, 21]. Furthermore, Ajuba proteins regulate JNK Hippo pathway activation by inhibiting LATS1/2 function [22]. More significantly, PI3K activation leads to Hippo pathway inhibition, conferring antagonism between contact inhibition and growth promotion [23].

\section{Materials and Methods}

Eligible articles were identified by a search of the MEDLINE bibliographical database for the last 5 years, i.e., the period of January 1, 2014 up to December 31, 2018. The search strategy included the following keywords: breast cancer [ti] AND (neoplasm [ti] OR cancer [ti] OR breast [ti]) AND (Hippo pathway [ti] OR YAP1 [ti] OR TAZ [ti]) AND (follow up [ti] OR prognosis [ti] OR treatment [ti]).

Language restrictions were applied (only articles in English, French, and German were considered eligible). Two investigators (F.Z. and A.K.), working independently, searched the literature and extracted data from each eligible study. Reviews, experts' opinions, as well as prospective and retrospective studies were eligible, while case reports were excluded for this systematic review. Manuscripts that did not state the name of the authors were excluded. Additional articles were identified from the reference lists of retrieved articles.

Articles with preclinical and clinical data were included. Literature referring to the biological role of the Hippo pathway in BC oncogenesis enriched our analysis. It must be highlighted that there was no limitation regarding the BC tumor subtype (ER, PR, Her2 status) of the reported articles. Publications reporting data from $\mathrm{BC}$ cell lines, animal models, patients' clinical samples, and in silico experiments are discussed. Especially, studies revealing prognostic relevance of Hippo pathway components in $\mathrm{BC}$ and studies therapeutically targeting Hippo signaling were considered eligible for this systematic review. 
Fig. 2. Schematic chart with the study design and the selection of articles included.

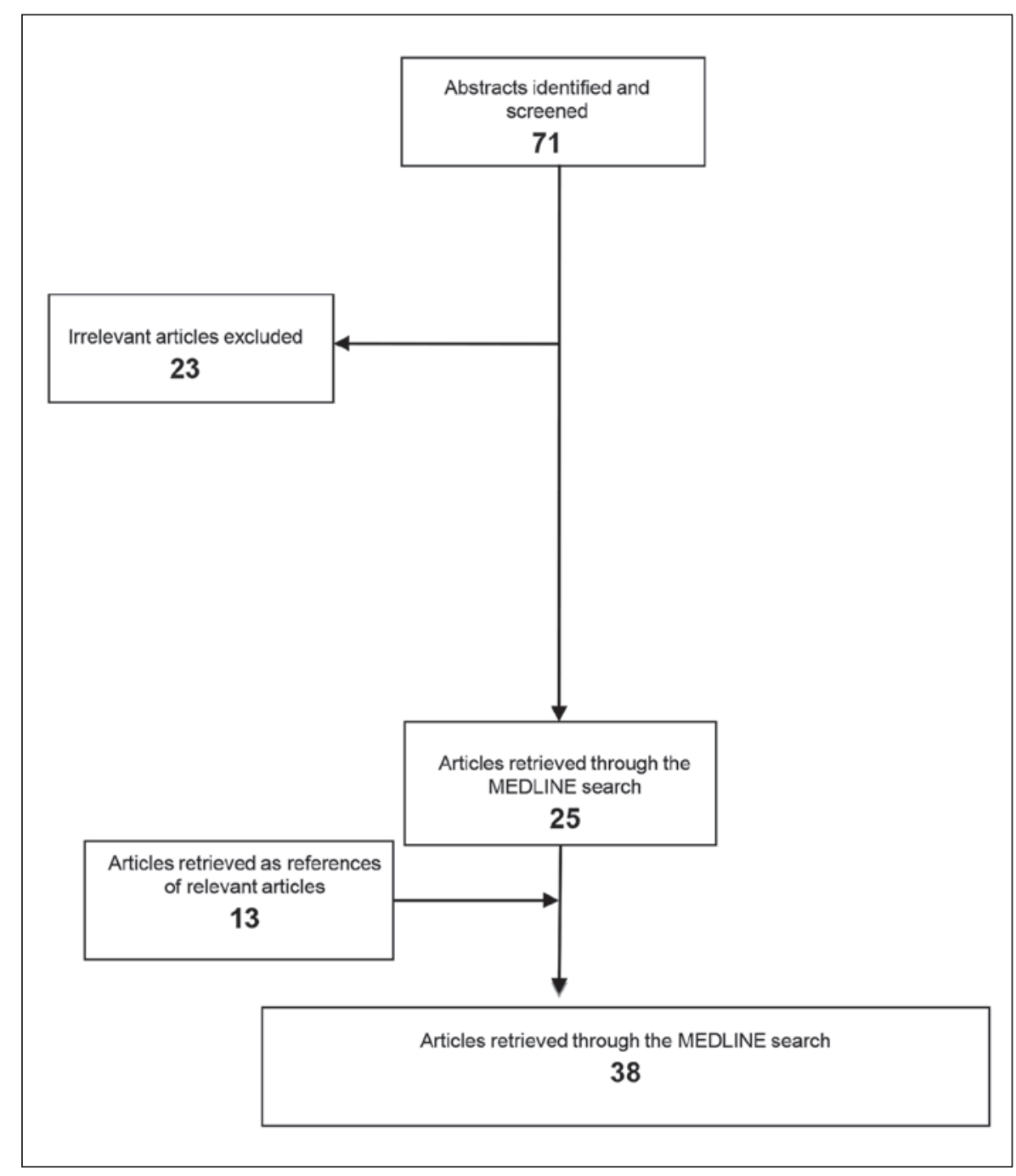

\section{Results}

Our research strategy revealed 71 articles, from which 23 were excluded. The remaining 48 articles included 6 reviews, 12 articles that described patient-derived data, 24 publications reporting data coming from cell lines, 5 papers discussing data derived both from patients and cell lines or xenografts or animals, and 1 paper which published data derived from animals. The MEDLINE research retrieved 25 relevant articles, while 13 papers were included being references of relevant articles (Fig. 2).

Both clinical and basic research articles were included. Studies regarding molecular mechanism associated with prognosis were performed on female cell lines or tumor samples $(n=21)[3,7,14,19,20,24-45]$ and male patient tumor samples $(n=3)$ [46-48]. More specifically, Hippo pathway implication in epithelial-mesenchymal transition (EMT), stem cell generation, and therapeutic resistance of BC cells were discussed. Furthermore, studies of the involvement of Hippo signaling elements in BC me- tastasis were included. Genetic variants data were extracted from patient samples $(n=2)[49,50]$.

New treatment options were studied on cell lines derived from $\mathrm{BC}$ patients and/or xenografts and genetically engineered animals $(n=13)[35,51-62]$. Verteporfin, apigenin, scutellarin, and bortezomib targeting Hippo signaling in BC are discussed. Additionally, Hippo pathway involvement in resistance to taxol and lapatinib and correlation to immunotherapy and CDK inhibition are reported.

Furthermore, computational analyses and bioinformatics data were also included [63]. Network signaling studies highlighting the Hippo pathway as an important cellular pathway are presented herein. Cross-talk of the Hippo pathway with several cell signalings, already known for their involvement in $\mathrm{BC}$, are highlighted, depicting the core position of the Hippo pathway in the oncogenic signal transduction of BC cells. The in silico data were completed by in vitro and in vivo experiments in order to functionally reevaluate the involvement of the Hippo pathway in $\mathrm{BC}$ tumorigenesis. 


\section{Discussion}

\section{The Hippo Pathway in BC Tumorigenesis and Prognosis}

Recent data showed that the Hippo pathway is frequently altered in several solid tumors, indicating a possible implication in their pathogenesis $[1,2]$. The crosstalk of the Hippo pathway via its several elements with other cellular signaling pathways makes it a promising drugable target [64]. The Hippo pathway is also engaged in $\mathrm{BC}$, promoting several functions such as EMT, stem cell generation, and therapeutic resistance $[65,66]$. Hippo signaling promotes $\mathrm{BC}$ metastasis [32], and its core elements play an important role in the molecular mechanism of colonization of BC cells either in or outside of breast tissue $[41,67]$.

YAP1 and TAZ expression is deregulated in BC [30, 31]. Elevated levels of YAP1 and TAZ cotranscription factors are reported in luminal $\mathrm{A}$ and luminal $\mathrm{B}, \mathrm{Her} 2+$, and triple-negative $\mathrm{BC}$ (TNBC) [65]. TAZ nuclear localization is associated with TNBC [24]. YAP1 and TAZ are also linked to metastasis in $\mathrm{BC}$ [68]. TAZ interacts with $\mathrm{HIF}-1 \alpha$ and is associated with bone metastasis in BC, as is shown from co-immunoprecipitation experiments [69]. Immunohistochemistry of HIF-1 $1 \alpha$ and TAZ in bone metastasis cells derived from BC parental cells revealed increased expression for both TAZ and HIF-1a [69]. Chromatin immunoprecipitation assays in MCF7 cells proved that TAZ acts as a coactivator of HIF-1a downstream targets and similarly HIF- $1 \alpha$ acts as a coactivator of TAZ target genes in human BC cells [27]. Furthermore, analysis of the human $\mathrm{BC}$ database revealed that increased patient mortality is associated with increased expression of both TAZ and HIF-1 target genes [70]. Decreased expression of upstream Hippo kinases LATS1/2 due to hypermethylation of LATS1/2 promoters has been reported in BC tumors with increased tumor size and lymph node metastases [71]. LATS1 expression was associated with disease outcome [72]. Low YAP1 expression has been reported as an independent prognostic factor of recurrence for luminal A BC patients [73]. More specifically, YAP1 protein intensity in $644 \mathrm{BC}$ tumor samples and YAP1 mRNA expression levels tested with microarrays from 1,104 BC patients showed that in estrogen receptor-positive $(\mathrm{ER}+)$ patients YAP1 is negatively correlated with proliferation and histological grade. On the other hand, in estrogen receptor-negative (ER-) patients YAP1 expression was correlated with proliferation measured by Ki67 [73]. Studies of ER- BC tumor samples showed that IL-6 increases YAP1 dephosphorylation, nuclear localization, and transcriptional activity, resulting in migration and invasion of BC cells [40]. This finding highlights the different roles that YAP1 plays among ER+ and ERBC subtypes. Furthermore, it depicts the complexity of the molecular mechanisms underlying YAP1's position and function in each case $[40,73]$.

In TNBC the MYC oncogene is overexpressed. In both $\mathrm{BC}$ cells and mouse models, MYC repressed YAP1 and TAZ. Further experiments showed that MYC deregulation suppressed TEAD transcription factor activity. YAP1 and TAZ are cotranscription factors mainly recruiting TEAD transcription factors to DNA. MYC, YAP1/TAZ, and TEAD circuitry may represent a critical pathway in the evolution of specific BC subtypes [28]. In mouse models MYC has been shown to suppress YAP1/TAZ activity in mammary tumors [26].

Furthermore, YAP1 is aberrantly expressed in TNBC. YAP1 and its upstream regulator Aurora kinase A localize predominantly in the nucleus of TNBC cells, implying a crucial role of the Hippo pathway in this BC subtype [74]. Co-immunoprecipitation studies, mass spectrometry, and expression analyses of both samples of TNBC patients and cell lines confirmed the interaction of Aurora kinase A with YAP1 and suggested that YAP1 is an important driver of malignant development in TNBC [74].

EMT is a cellular dedifferentiation program which gives an aggressive phenotype to cells through several ways. It confers cells with stem cell-like characteristics, promotes metastasis and tumor invasion, and makes malignant cells resistant to chemotherapy. In BC cells, EMT activation via Twist overexpression suppresses the Hippo pathway. In detail, Twist-overexpressing luminal BC cells show significant increase in Par1 activity - an upstream regulator of Hippo - which in turn interacts with TAZ by increasing its expression and deregulating the normal Hippo pathway [33]. Elevated expression of CTGF, a downstream target of the Hippo pathway, accompanies Twist-mediated EMT. Wound healing and invasiveness experiments confirmed that Twist-mediated EMT via Hippo inhibition promoted invasiveness and increased the migratory ability of BC cells [33]. Furthermore, Notch3 inhibits EMT through the Hippo pathway by activating Kibra. Chromatin immunoprecipitation experiments revealed that Notch3 interacts directly with Kibra by binding to the RBP-Jk-binding site of the latter, thus activating Kibra protein. Knockdown of Kibra in BC epithelial cells reversed EMT [34]. These mechanisms are important for $\mathrm{BC}$ evolution and reveal new therapeutic targets and strategies regarding $\mathrm{BC}$ invasion and metastasis.

The involvement of the Hippo pathway in BC stem cells (BCSCs) was first described by Cordenonsi et al. in 2011 [63], and today this involvement is very well documented $[75,76]$. Microarray test and computational analysis of 993 primary tumors revealed a YAP1/TAZ signature to be enriched in poorly differentiated $\mathrm{BC}$ tumors [63]. TAZ is highly expressed in grade 3 compared to grade 1 tumors arising in immunocompromised mice injected with isogenic derivative cells of the MFC10A cell 
line, depicting that TAZ is required for self-renewal and tumor initiation of BC cells [63]. Further studies in human primary $\mathrm{BC}$ confirmed that TAZ-positive tumors are associated with grade 3 disease [63]. Several publications report that TAZ activation enhances the metastatic potential of BCSCs and TNBC through different mechanisms $[25,77,78]$. In basal-like BC cells, TAZ-dependent molecular signals were identified [45]. Expression analysis of 99 tumor samples from BC patients with immunohistochemistry for TAZ revealed that high expression of TAZ was an independent negative marker for diseasefree survival [77]. Expression studies in 59 primary tumors with their paired metachronous metastases, combined with further studies in BCSC xenografts paired with their tumor metastases, showed a clear increase in TAZ expression in the metastatic sites [77]. Suppression of TAZ is facilitated by Ski with several biological mechanisms. Ski activates LATS2 kinase, which phosphorylates TAZ and subsequently leads to TAZ degradation [25]. Additionally, Ski binds to TEAD and inhibits TAZ transcriptional activity by recruiting cotranscription repressor NCoR1 [25]. Therefore, Ski antagonizes TAZ-induced EMT in BC cell lines [25].

In TNBC cell lines, apigenin is shown to decrease YAP1/TAZ activity and therefore reduces the expression of Hippo pathway downstream target genes, such as Cyr61 and CTGF. Apigenin disrupts the YAP1/TAZTEAD interaction and thus downregulates the expression of Hippo downstream targets. Targeting the Hippo pathway with apigenin is a promising way to potentially overcome resistance to chemotherapy and metastasis [79]. Here, it should be noted that although apigenin has antitumor activity, experiments in erythroleukemia cell lines have shown that it blocks cell cycle and induction of autophagy, which resulted in lower levels of response to vincristine [80].

Further studies on TNBC patient tumor samples and xenografts have shown that synaptopodin-2 inhibits YAP1 and TAZ activity and inhibits invasion and metastasis [42]. In TNBC cells, knocking down TAZ results in increased sensitivity to EGFR inhibitors, thus providing evidence for the potential explanation of the low effectiveness of anti-EGFR therapy [29].

On the other hand, YAP1 appears to have both tumorsuppressing and tumor-promoting properties [81, 82]. Array CGH data from mice and mammary tumors revealed an amplicon on 11q22 pointing to the YAP1 gene. YAP1-overexpressing cells showed EMT, proliferative advantage, and inhibition of apoptosis showing an oncogenic function in mammalian cells [81]. Tumor suppressor properties have been described for YAP1 in BC cells [83]. Inhibition of YAP1 expression with microRNA200a in BC cells promoted anoikis resistance and metastasis [84]. Loss of DLG5 promotes BC cell proliferation by increasing the nuclear levels of YAP1 [37]. Kim and Lim [85] reported that serum response factor binds YAP1 and recruits it to target genes sharing mammary stem cell-like characteristics. Serum response factor, YAP1, and IL- 6 are overexpressed in basal-like and TNBC cells and are correlated with BCSC generation and poor relapse-free survival, specifically in basal-like BC [85].

Drug tests in TNBC cell lines revealed that synthetic glucocorticoids were the compounds with the strongest effect on increasing YAP1 protein levels. Treatment of TNBC cells with glucocorticoids also increased YAP's downstream targets, such as CTGF and Cyr61. Moreover, the glucocorticoid receptor activated YAP1 via fibronectin 1 , as was shown from chromatin immunoprecipitation studies, proposing a cytoskeleton remodeling-dependent manner of activation of the Hippo pathway [38]. Studies in patient-derived samples, including primary tumors and metastatic lymph nodes, revealed a YAP1/ THBS1/FAK axis that regulates tumor invasiveness and focal adhesion [43]. All these together highlight the important cross-talk of the Hippo pathway with cytoskeletal regulation and mechanical influence.

RASSF6 in BC regulates the Hippo pathway. More specifically, immunohistochemical studies of RASSF6 in 95 BC tumor samples revealed low expression of RASSF6 in 42 cases. Especially in TNBC, RASSF6 downregulation was stronger [39]. RASSF6 downregulates YAP1 by upregulating MST1/2 and LATS1 phosphorylation, depicting a direct association of RASSF6 with Hippo signaling during $\mathrm{BC}$ tumorigenesis [39]. Epigenetic regulation of RASSF1A through promoter regulation reduces pYAP and prevents invasion in BC tumors [86]. Furthermore, RASSF1A through TGF- $\beta$ regulates YAP1 transcriptional activity [87]. All these data highlight the important role of RASSF proteins as master regulators of the Hippo pathway, although the potential prognostic significance of these molecular mechanisms in selecting the patients that might benefit from therapeutic approaches targeting the Hippo pathway still remains unclear.

MARK4 can bind to MST1/2-SAV and inhibits the complex formation between LATS1/2, MST1/2, and SAV and therefore negatively regulates YAP1 and TAZ [36]. Further studies in BC cells showed that MARK4 depletion facilitated proliferation and migration [36]. Additionally, MAC30 was found to be upregulated in BC cells. Increased levels of phosphorylated YAP1, MST1, and LATS1 were discovered after MAC30 knockout with siRNA in BC cells, revealing that MAC30 knockdown results in Hippo pathway activation [44].

Another biological process which interacts with the Hippo pathway was recently reported [88]. Geranylgeranylation is a lipidation process conjugating geranylgeranyl to the carboxyl terminal CAAX motif of proteins for anchoring to membranes. Inhibition of geranylgeranyl 
pyrophosphate with atorvastatin or of geranylgeranylation with inhibitor GGTI-298 in BC cells resulted in an increase of MST1/2 and LATS1 phosphorylation, thus upregulating the Hippo pathway [88]. Moreover, BC cell proliferation and migration were dependent on geranylgeranylation-mediated activation of the Hippo pathway $[88,89]$.

Male $\mathrm{BC}$ is a rare disease, with our understanding on its biology still being vague [46]. The Hippo pathway regulators YAP1 and TAZ and their downstream target CTGF were studied in 129 patients. Patients sharing the YAP1/CTGF and TAZ/CTGF phenotypes had a shorter overall survival. Statistical analysis indicated that these phenotypes are independent predictors of survival [47]. Further studies of 116 male BC samples revealed that AXL expression is associated with overall survival. More significantly, phenotypes YAP1/CTGF/AXL and TAZ/ CTGF/AXL had an inferior survival compared to nontriple-positive phenotypes [48].

Genetic variances of Hippo pathway genes have been tested in African American and African populations. It was shown that the Hippo pathway was associated with risk of ER- BC in the genetic variation analysis of 3,663 cases (1,983 ER+ and 1,098 ER-) paired with 4,687 controls. The $\mathrm{CDH} 1$ gene variant was connected to ER- BC in African American women [49]. On the other hand, the Hippo pathway was associated with risk of $\mathrm{ER}+\mathrm{BC}$ in a female population of African ancestry. The WWC1 (KIBRA) gene was the most statistically significant single nucleotide polymorphism in an African-oriented population including $403 \mathrm{ER}+$ and $374 \mathrm{ER}$ - women out of 1,657 cases examined for 7,086 single nucleotide polymorphisms [50].

\section{The Hippo Pathway and Systemic Treatment}

Hippo pathway deregulation has been implicated in resistance to chemotherapeutic drugs [63]. The Hippo pathway can be used as a target for therapeutic intervention [61]. Small molecules like verteporfin, statins, and bisphosphonates are being evaluated as putative treatment options [52, 55, 57]. Bortezomib, a proteasome inhibitor, is being evaluated in phase III trials including Bcell non-Hodgkin lymphoma, acute myelogenous leukemia, multiple myeloma, prostate cancer, and advanced soft tissue sarcomas [57]. The molecular mechanism by which bortezomib interacts with the Hippo pathway involves FOXM1 [90]. FOXM1 is amplified in BC and is highly enriched in basal-type BCs. FOXM1 upregulates Aurora kinase and activates the Hippo pathway. Furthermore, FOXM1 is upregulated by YAP1-TEAD transcription [90]. Bortezomib suppresses FOXM1 and induces apoptosis [91].

Cyr61 and CTGF, two downstream targets of TAZ/ TEAD, are associated with paclitaxel resistance in $\mathrm{BC}$ cells. Inhibition of Cyr61 and CTGF with shRNA technology reversed the response to paclitaxel [51]. TAZ has been shown to mediate resistance to paclitaxel in BC cells [51]. Further experiments with microarray in tissue samples from patients treated with chemotherapeutic regimens such as taxol could examine the predictive value of TAZ as a biomarker in drug response [51]. Tamoxifen resistance via TAZ overexpression in BCSCs with low DLG5 levels indicates a molecular mechanism by which the Hippo pathway is involved in resistance and stemness [62]. Dasatinib has been shown to sensitize TNBC cells to paclitaxel and help overcome paclitaxel resistance [78]. This function is performed through downregulating Src kinase [92].

Neoadjuvant therapy responsiveness is also correlated with the Hippo pathway. Biopsies from Her2+ and TNBC patients who received neoadjuvant therapy were tested for the Hippo pathway elements' localization and expression. Nuclear localization of kinases MST1/2 affects the efficacy of neoadjuvant therapy in BC and is associated with worse outcome. MST1/2 are incorporated to DNA damage response through cooperation with the ATR and ATM pathways and create molecular interactions of chemoresistance $[56,58]$. In luminal B and HER2-positive BC patients, the TAZ score predicts pathological complete response to neoadjuvant chemotherapy and trastuzumab [53].

In TNBC cells, YAP1 has been shown to be upregulated. mRNA levels of YAP1 in 4,000 BC patients failed to show association with relapse-free survival, but when the analysis was restricted to TNBC patients, YAP1 expression correlated with decreased relapse-free survival [35]. Inhibition of YAP1 in TNBC cells either genetically (siRNA and shRNA) or pharmacologically with verteporfin resulted in radiosensitization. In detail, YAP mediates its radioresistance via PI3K/AKT and EGFR survival signaling depicted in the MDA-MB-231 TNBC cell line. DNA-damaging modalities targeting the Hippo pathway may represent a new therapeutic approach for TNBC [35].

Progression in Her2+ tumors is a phenomenon not well understood, including changes in molecular pathways and tumor microenvironment. Lapatinib is a Her2 kinase inhibitor. Resistance to lapatinib has been connected to changes in the biophysical properties of tumor tissue due to extracellular matrix rigidity. The Hippo pathway is deregulated through mechanotransduction resulting in YAP1 and TAZ overexpression, relocation of YAP1 and TAZ in the nucleus, and lapatinib resistance. Inhibition of the Hippo pathway, either genetically or pharmacologically with verteporfin, reversed the response to lapatinib in BC cells implanted in mice [54].

Experiments in $\mathrm{BC}$ cells showed a direct association of HMGA1, CCNE2, and YAP. More precisely, HMGA1 and CCNE2 regulate YAP1 phosphorylation and subse- 
quent nuclear localization. Further studies with CDK inhibitors affected YAP1 nuclear localization. Additionally, YAP1's downstream targets such as CTGF and Cyr61 were downregulated when $\mathrm{BC}$ cells were treated with CDK inhibitors [57].

The Hippo pathway has also been connected with immune evasion. In BC cell lines and mouse cell lines tested with Nanostring gene expression profiling, PD-L1 was found to be the target of YAP1 and TAZ cotranscription factors. TAZ/YAP1 via TEAD activate PD-L1 promoter, thus causing inhibition of T cells [60]. These data suggest the therapeutic potential for BC treatment in targeting the Hippo pathway either as a monotherapy or in combination with PD-L1-targeted immunotherapy [60].

Scutellarin is an effective component of the Scutellaria barbata herb, which has been shown to have antitumor activity in hepatocellular carcinoma, colon cancer, and tongue squamous cell carcinoma. Tests of scutellarin in MCF7 BC cells and xenografts showed that scutellarintreated cells presented with lower growth and increased apoptosis through regulation of the Hippo pathway. More specifically, increased pYAP and lower YAP1 expression was associated with inhibition of tumor growth in vivo in scutellarin-treated mice [59]. The exact molecular mechanism of scutellarin regulating pYAP and YAP1 expression is not well understood. Furthermore, it must be noted that scutellarin has not yet been tested in humans and that only preclinical data support its effectiveness as a therapeutic approach.

\section{Conclusion and Future Challenges}

The Hippo pathway is a recently described pathway which has several oncogenic functions. In BC this pathway is involved in oncogenesis and tumor evolution [65]. Especially in TNBC, where the driving molecular pathways are still unknown, the Hippo pathway seems to play a crucial role [56].

Cross-talk of the Hippo pathway with other molecular pathways gives a compound position of Hippo signaling in BC tumor cells. PI3K/AKT, Notch, and EGFR signaling are common Hippo partners conferring to the complexity of the oncogenic process. Thus, EMT, invasiveness, metastatic potential, and stem cell characteristics are tumor characteristics that $\mathrm{BC}$ cells can exhibit when the Hippo pathway is deregulated [33, 67].

Hippo molecular elements - YAP1, TAZ, Kibra, Aurora kinase, and LATS - have been shown to participate in $\mathrm{BC}$ development through different mechanisms [65, 69, 71, 73]. YAP1 and TAZ expression is associated with luminal A and luminal B, Her2+, and TNBC [65]. It must be pointed out that YAP1 has different roles among different BC subtypes. Especially for ER+ BC, YAP1 corre- lates negatively with tumor grade and proliferation, whereas on the other hand in ER- BC, it correlates positively with proliferation. TAZ expression is related to metastasis and hypoxia [69]. EMT and BCSC-like characteristics are related to Kibra and TAZ [33, 34].

Response to systemic therapy, including all existing options - chemotherapy, immunotherapy, and radiation therapy - has also been associated with Hippo signaling deregulation. TAZ is examined as a predictive biomarker for drug response [77]. Resistance to systemic treatment used in BC such as paclitaxel, or lapatinib that targets Her2 signaling, can be reversed by inhibiting Hippo pathway elements [51, 54]. CDK inhibitors induce the translocation of YAP1 from the nucleus to the cytoplasm, resulting in a decrease in its activity [57]. Insights into immunotherapy link YAP1 and TAZ transcription cofactors via TEAD to PD-L1 and immune evasion, raising crucial questions regarding the potential use of Hippo-targeted therapy alone or in combination with immunotherapy [60].

The Hippo pathway can be a target to reverse resistance to systemic treatments. Small-molecule verteporfin is used at experimental levels both in vitro and in vivo to inhibit the Hippo pathway [35]. Bortezomib, a proteasome inhibitor which is already clinically used in multiple myeloma, is currently studied in several solid tumors. Interaction of Hippo signaling with molecules showing antitumor activity, such as scutellarin, may lead to new therapeutic modalities in BC [59].

The Hippo pathway has a pivotal role in $\mathrm{BC}$ molecular biology, cellular behavior, and response to treatment. Especially through its cross-talk with other oncogenic signaling pathways and biological processes it becomes a promising drugable target. However, it should be highlighted that BC patients who are going to benefit from therapies targeting the Hippo pathway are not yet well defined. Our understanding on this signaling pathway will provide scientists and clinicians with important information regarding $\mathrm{BC}$ tumorigenesis and development. Moreover, targeting Hippo signaling may prove to be an important weapon for future therapeutic interventions in the armamentarium of clinicians dealing with BC.

\section{Statement of Ethics}

The authors declare that this study was conducted according to the laws of Greece. It was conducted and reported in accordance with the Preferred Reporting Items for Systematic Reviews and Meta-Analyses statement. The protocol of this systematic review was submitted to the Institutional Review Board of Alexandra Hospital, Medical University of Athens, Greece. This article does not contain any studies with human participants performed by any of the authors.
Kyriazoglou et al. 


\section{Disclosure Statement}

F. Zagouri has received honoraria from Roche, Novartis, and Lilly.

\section{Funding Sources}

No funding was offered.

\section{Author Contributions}

F. Zagouri and A. Kyriazoglou were the writers of the article, were the two independent investigators who performed the literature search and data extraction from all studies examined, and contributed to the conception and design of the study. Data acquisition was done by A. Kyriazoglou, M. Kaparelou, R. Georgakopoulou, A. Tsiara, A.M. Papatheodoridi, and R. Zakopoulou. Manuscript editing and revision were performed by M. Liontos and F. Zagouri. All authors read and approved the final manuscript.

\section{References}

1 Varelas X. The Hippo pathway effectors TAZ and YAP in development, homeostasis and disease. Development. 2014 Apr;141(8): 1614-26.

2 Zhang K, Qi HX, Hu ZM, Chang YN, Shi ZM, $\mathrm{Han} \mathrm{XH}$, et al. YAP and TAZ Take Center Stage in Cancer. Biochemistry. 2015 Nov; 54(43):6555-66.

3 Britschgi A, Duss S, Kim S, Couto JP, Brinkhaus H, Koren S, et al. The Hippo kinases LATS1 and 2 control human breast cell fate via crosstalk with ERa. Nature. 2017 Jan; 541(7638):541-5.

4 Stein C, Bardet AF, Roma G, Bergling S, Clay I, Ruchti A, et al. YAP1 Exerts Its Transcriptional Control via TEAD-Mediated Activation of Enhancers. PLoS Genet. 2015 Aug; 11(8):e1005465.

5 Ehmer U, Sage J. Control of Proliferation and Cancer Growth by the Hippo Signaling Pathway. Mol Cancer Res. 2016 Feb;14(2):127-40.

6 Yu FX, Zhao B, Panupinthu N, Jewell JL, Lian I, Wang LH, et al. Regulation of the HippoYAP pathway by G-protein-coupled receptor signaling. Cell. 2012 Aug;150(4):780-91.

7 Zhou X, Wang S, Wang Z, Feng X, Liu P, Lv $\mathrm{XB}$, et al. Estrogen regulates Hippo signaling via GPER in breast cancer. J Clin Invest. 2015 May;125(5):2123-35.

8 Avruch J, Praskova M, Ortiz-Vega S, Liu M, Zhang XF. Nore1 and RASSF1 regulation of cell proliferation and of the MST1/2 kinases. Methods Enzymol. 2006;407:290-310.

9 Yu J, Zheng Y, Dong J, Klusza S, Deng WM, Pan D. Kibra functions as a tumor suppressor protein that regulates Hippo signaling in conjunction with Merlin and Expanded. Dev Cell. 2010 Feb;18(2):288-99.

10 Kim S, Jho EH. Merlin, a regulator of Hippo signaling, regulates $\mathrm{Wnt} / \beta$-catenin signaling. BMB Rep. 2016 Jul;49(7):357-8.

11 Halder G, Dupont S, Piccolo S. Transduction of mechanical and cytoskeletal cues by YAP and TAZ. Nat Rev Mol Cell Biol. 2012 Sep; 13(9):591-600.

12 Rauskolb C, Sun S, Sun G, Pan Y, Irvine KD. Cytoskeletal tension inhibits Hippo signaling through an Ajuba-Warts complex. Cell. 2014 Jul;158(1):143-56.

13 Fan R, Kim NG, Gumbiner BM. Regulation of Hippo pathway by mitogenic growth factors via phosphoinositide 3-kinase and phosphoinositide-dependent kinase-1. Proc Nat Acad Sci USA. 2013 Feb;110(7):2569-74.
14 Hiemer SE, Szymaniak AD, Varelas X. The transcriptional regulators TAZ and YAP direct transforming growth factor $\beta$-induced tumorigenic phenotypes in breast cancer cells. J Biol Chem. 2014 May;289(19):1346174.

15 Piersma B, Bank RA, Boersema M. Signaling in Fibrosis: TGF- $\beta$, WNT, and YAP/TAZ Converge. Front Med (Lausanne). 2015 Sep;2: 59.

16 Zanconato F, Forcato M, Battilana G, Azzolin L, Quaranta E, Bodega B, et al. Genome-wide association between YAP/TAZ/TEAD and AP-1 at enhancers drives oncogenic growth. Nat Cell Biol. 2015 Sep;17(9):1218-27.

17 Cheng F, Zhao J, Hanker AB, Brewer MR, Arteaga CL, Zhao Z. Transcriptome- and proteome-oriented identification of dysregulated eIF4G, STAT3, and Hippo pathways altered by PIK3CA H1047R in HER2/ER-positive breast cancer. Breast Cancer Res Treat. 2016 Dec;160(3):457-74.

18 Lin L, Bivona TG. The Hippo effector YAP regulates the response of cancer cells to MAPK pathway inhibitors. Mol Cell Oncol. 2015 Apr;3(1):e1021441.

19 Zhu Q, Le Scolan E, Jahchan N, Ji X, Xu A, Luo K. SnoN Antagonizes the Hippo Kinase Complex to Promote TAZ Signaling during Breast Carcinogenesis. Dev Cell. 2016 Jun; 37(5):399-412.

20 Samanta S, Guru S, Elaimy AL, Amante JJ, Ou J, Yu J, et al. IMP3 Stabilization of WNT5B mRNA Facilitates TAZ Activation in Breast Cancer. Cell Rep. 2018 May;23(9):2559-67.

21 Liu X, Li H, Rajurkar M, Li Q, Cotton JL, Ou J, et al. Tead and AP1 Coordinate Transcription and Motility. Cell Rep. 2016 Feb;14(5): 1169-80.

22 Sun G, Irvine KD. Ajuba family proteins link JNK to Hippo signaling. Sci Signal. 2013 Sep; 6(292):ra81.

23 Huang W, Lv X, Liu C, Zha Z, Zhang H, Jiang $\mathrm{Y}$, et al. The N-terminal phosphodegron targets TAZ/WWTR1 protein for SCF $\beta$-TrCPdependent degradation in response to phosphatidylinositol 3-kinase inhibition. J Biol Chem. 2012 Jul;287(31):26245-53.

24 Díaz-Martín J, López-García MA, RomeroPérez L, Atienza-Amores MR, Pecero ML, Castilla MA, et al. Nuclear TAZ expression associates with the triple-negative phenotype in breast cancer. Endocr Relat Cancer. 2015 Jun; 22(3):443-54
25 Rashidian J, Le Scolan E, Ji X, Zhu Q, Mulvihill MM, Nomura D, et al. Ski regulates Hippo and TAZ signaling to suppress breast cancer progression. Sci Signal. 2015 Feb; 8(363):ra14.

26 von Eyss B, Jaenicke LA, Kortlever RM, Royla $\mathrm{N}$, Wiese KE, Letschert S, et al. A MYC-Driven Change in Mitochondrial Dynamics Limits YAP/TAZ Function in Mammary Epithelial Cells and Breast Cancer. Cancer Cell. 2015 Dec;28(6):743-57.

27 Xiang L, Gilkes DM, Hu H, Luo W, Bullen JW, Liang H, et al. HIF-1 $\alpha$ and TAZ serve as reciprocal co-activators in human breast cancer cells. Oncotarget. 2015 May;6(14):1176878.

28 Elster D, Jaenicke LA, Eilers M, von Eyss B. TEAD activity is restrained by MYC and stratifies human breast cancer subtypes. Cell Cycle. 2016 Oct;15(19):2551-6.

29 Guo L, Zheng J, Zhang J, Wang H, Shao G, Teng L. Knockdown of TAZ modifies triplenegative breast cancer cell sensitivity to EGFR inhibitors by regulating YAP expression. Oncol Rep. 2016 Aug;36(2):729-36.

30 Liu CY, Chan SW, Guo F, Toloczko A, Cui L, Hong W. MRTF/SRF dependent transcriptional regulation of TAZ in breast cancer cells. Oncotarget. 2016 Mar;7(12):13706-16.

31 Sun JG, Chen XW, Zhang LP, Wang J, Diehn M. Yap1 promotes the survival and self-renewal of breast tumor initiating cells via inhibiting Smad3 signaling. Oncotarget. 2016 Mar;7(9):9692-706.

32 Wang J, Rouse C, Jasper JS, Pendergast AM. ABL kinases promote breast cancer osteolytic metastasis by modulating tumor-bone interactions through TAZ and STAT5 signaling. Sci Signal. 2016 Feb;9(413):ra12.

33 Wang Y, Liu J, Ying X, Lin PC, Zhou BP. Twist-mediated Epithelial-mesenchymal Transition Promotes Breast Tumor Cell Invasion via Inhibition of Hippo Pathway. Sci Rep. 2016 Apr;6(1):24606.

34 Zhang X, Liu X, Luo J, Xiao W, Ye X, Chen M, et al. Notch3 inhibits epithelial-mesenchymal transition by activating Kibra-mediated Hippo/YAP signaling in breast cancer epithelial cells. Oncogenesis. 2016 Nov;5(11):e269.

35 Andrade D, Mehta M, Griffith J, Panneerselvam J, Srivastava A, Kim TD, et al. YAP1 inhibition radiosensitizes triple negative breast cancer cells by targeting the DNA damage response and cell survival pathways. Oncotarget. 2017 Oct;8(58):98495-508. 
36 Heidary Arash E, Shiban A, Song S, Attisano L. MARK4 inhibits Hippo signaling to promote proliferation and migration of breast cancer cells. EMBO Rep. 2017 Mar;18(3):420-36.

37 Liu J, Li J, Li P, Wang Y, Liang Z, Jiang Y, et al. Loss of DLG5 promotes breast cancer malignancy by inhibiting the Hippo signaling pathway. Sci Rep. 2017 Feb;7(1):42125.

38 Sorrentino G, Ruggeri N, Zannini A, Ingallina E, Bertolio R, Marotta C, et al. Glucocorticoid receptor signalling activates YAP in breast cancer. Nat Commun. 2017 Jan;8(1):14073.

39 He Z, Zhao TT, Jin F, Li JG, Xu YY, Dong HT, et al. Downregulation of RASSF6 promotes breast cancer growth and chemoresistance through regulation of Hippo signaling. Biochem Biophys Res Commun. 2018 Sep; 503(4):2340-7.

40 Hou L, Xie S, Li G, Xiong B, Gao Y, Zhao X, et al. IL-6 Triggers the Migration and Invasion of Oestrogen Receptor-Negative Breast Cancer Cells via Regulation of Hippo Pathways. Basic Clin Pharmacol Toxicol. 2018 Nov;123(5):549-57.

41 Li SY, Wang H, Mai HF, Li GF, Chen SJ, Li GS, et al. Down-regulated long non-coding RNA RNAZFHX4-AS1 suppresses invasion and migration of breast cancer cells via FAT4dependent Hippo signaling pathway. Cancer Gene Ther. 2019 Nov;26(11-12):374-87.

42 Liu J, Ye L, Li Q, Wu X, Wang B, Ouyang Y, et al. Synaptopodin-2 suppresses metastasis of triple-negative breast cancer via inhibition of YAP/TAZ activity. J Pathol. 2018 Jan;244(1): 71-83.

43 Shen J, Cao B, Wang Y, Ma C, Zeng Z, Liu L, et al. Hippo component YAP promotes focal adhesion and tumour aggressiveness via transcriptionally activating THBS1/FAK signalling in breast cancer. J Exp Clin Cancer Res. 2018 Jul;37(1):175.

44 Song GQ, Zhao Y. MAC30 knockdown involved in the activation of the Hippo signaling pathway in breast cancer cells. Biol Chem. 2018 Oct;399(11):1305-11.

45 Shen H, Yang N, Truskinovsky A, Chen Y, Mussell AL, Nowak NJ, et al. Targeting TAZDriven Human Breast Cancer by Inhibiting a SKP2-p27 Signaling Axis. Mol Cancer Res. 2019 Jan;17(1):250-62.

46 Di Benedetto A, Mottolese M, Sperati F, Ercolani C, Di Lauro L, Pizzuti L, et al. HMGCoAR expression in male breast cancer: relationship with hormone receptors, Hippo transducers and survival outcomes. Sci Rep. 2016 Oct;6(1):35121.

47 Di Benedetto A, Mottolese M, Sperati F, Ercolani C, Di Lauro L, Pizzuti L, et al. The Hippo transducers TAZ/YAP and their target CTGF in male breast cancer. Oncotarget. 2016 Jul;7(28):43188-98.

48 Di Benedetto A, Mottolese M, Sperati F, Ercolani C, Di Lauro L, Pizzuti L, et al. Association between AXL, Hippo Transducers, and Survival Outcomes in Male Breast Cancer. J Cell Physiol. 2017 Aug;232(8):2246-52.

49 Zhang J, Yao S, Hu Q, Zhu Q, Liu S, Lunetta $\mathrm{KL}$, et al. Genetic variations in the Hippo signaling pathway and breast cancer risk in African American women in the AMBER Consortium. Carcinogenesis. 2016 Oct;37(10):9516.
50 Wang S, Huo D, Ogundiran TO, Ojengbede $\mathrm{O}$, Zheng W, Nathanson KL, et al. Genetic variation in the Hippo pathway and breast cancer risk in women of African ancestry. Mol Carcinog. 2018 Oct;57(10):1311-8.

51 Lai D, Ho KC, Hao Y, Yang X. Taxol resistance in breast cancer cells is mediated by the hippo pathway component TAZ and its downstream transcriptional targets Cyr61 and CTGF. Cancer Res. 2011 Apr;71(7): 2728-38.

52 Park HW, Guan KL. Regulation of the Hippo pathway and implications for anticancer drug development. Trends Pharmacol Sci. 2013 Oct;34(10):581-9.

53 Vici P, Mottolese M, Pizzuti L, Barba M, Sperati F, Terrenato I, et al. The Hippo transducer TAZ as a biomarker of pathological complete response in HER2-positive breast cancer patients treated with trastuzumab-based neoadjuvant therapy. Oncotarget. 2014 Oct; 5(20):9619-25.

54 Lin CH, Pelissier FA, Zhang H, Lakins J, Weaver VM, Park C, et al. Microenvironment rigidity modulates responses to the HER2 receptor tyrosine kinase inhibitor lapatinib via YAP and TAZ transcription factors. Mol Biol Cell. 2015 Nov;26(22):3946-53.

55 Oku Y, Nishiya N, Shito T, Yamamoto R, Yamamoto Y, Oyama C, et al. Small molecules inhibiting the nuclear localization of YAP/ TAZ for chemotherapeutics and chemosensitizers against breast cancers. FEBS Open Bio. 2015 Jun;5(1):542-9.

56 Vici P, Ercolani C, Di Benedetto A, Pizzuti L, Di Lauro L, Sperati F, et al. Topographic expression of the Hippo transducers TAZ and YAP in triple-negative breast cancer treated with neoadjuvant chemotherapy. J Exp Clin Cancer Res. 2016 Apr;35(1):62.

57 Ye S, Eisinger-Mathason TS. Targeting the Hippo pathway: clinical implications and therapeutics. Pharmacol Res. 2016 Jan;103: 270-8.

58 Ercolani C, Di Benedetto A, Terrenato I, Pizzuti L, Di Lauro L, Sergi D, et al. Expression of phosphorylated Hippo pathway kinases (MST1/2 and LATS1/2) in HER2-positive and triple-negative breast cancer patients treated with neoadjuvant therapy. Cancer Biol Ther. 2017 May;18(5):339-46.

59 Hou L, Chen L, Fang L. Scutellarin Inhibits Proliferation, Invasion, and Tumorigenicity in Human Breast Cancer Cells by Regulating HIPPO-YAP Signaling Pathway. Med Sci Monit. 2017 Oct;23:5130-8.

60 Janse van Rensburg HJ, Azad T, Ling M, Hao Y, Snetsinger B, Khanal P, et al. The Hippo Pathway Component TAZ Promotes Immune Evasion in Human Cancer through PD-L1. Cancer Res. 2018 Mar;78(6):145770.

61 Wu L, Yang X. Targeting the Hippo Pathway for Breast Cancer Therapy. Cancers (Basel). 2018 Nov; 10(11):E422.

62 Liu J, Li J, Li P, Jiang Y, Chen H, Wang R, et al. DLG5 suppresses breast cancer stem celllike characteristics to restore tamoxifen sensitivity by inhibiting TAZ expression. J Cell Mol Med. 2019 Jan;23(1):512-21.
63 Cordenonsi M, Zanconato F, Azzolin L, Forcato M, Rosato A, Frasson C, et al. The Hippo transducer TAZ confers cancer stem cell-related traits on breast cancer cells. Cell. 2011 Nov;147(4):759-72.

64 Yao CB, Zhou X, Chen CS, Lei QY. The regulatory mechanisms and functional roles of the Hippo signaling pathway in breast cancer. Yi Chuan. 2017 Jul;39(7):617-29.

65 Maugeri-Saccà M, Barba M, Pizzuti L, Vici P, Di Lauro L, Dattilo R, et al. The Hippo transducers TAZ and YAP in breast cancer: oncogenic activities and clinical implications. Expert Rev Mol Med. 2015 Jul;17:e14.

66 Shi P, Feng J, Chen C. Hippo pathway in mammary gland development and breast cancer. Acta Biochim Biophys Sin (Shanghai). 2015 Jan;47(1):53-9.

67 Wei C, Wang Y, Li X. The role of Hippo signal pathway in breast cancer metastasis. Onco Targets Ther. 2018 Apr;11:2185-93.

68 Zheng L, Xiang C, Li X, Guo Q, Gao L, Ni H, et al. STARD13-correlated ceRNA networkdirected inhibition on YAP/TAZ activity suppresses stemness of breast cancer via coregulating Hippo and Rho-GTPase/F-actin signaling. J Hematol Oncol. 2018 May;11(1): 72 .

69 Bendinelli P, Maroni P, Matteucci E, Luzzati A, Perrucchini G, Desiderio MA. Hypoxia inducible factor- 1 is activated by transcriptional co-activator with PDZ-binding motif (TAZ) versus WWdomain-containing oxidoreductase (WWOX) in hypoxic microenvironment of bone metastasis from breast cancer. Eur J Cancer. 2013 Jul;49(11):260818.

70 Xiang L, Gilkes DM, Hu H, Takano N, Luo W $\mathrm{Lu} \mathrm{H}$, et al. Hypoxia-inducible factor 1 mediates TAZ expression and nuclear localization to induce the breast cancer stem cell phenotype. Oncotarget. 2014 Dec;5(24):12509-27.

71 Visser S, Yang X. LATS tumor suppressor: a new governor of cellular homeostasis. Cell Cycle. 2010 Oct;9(19):3892-903.

72 Takahashi Y, Miyoshi Y, Takahata C, Irahara N, Taguchi T, Tamaki Y, et al. Down-regulation of LATS1 and LATS2 mRNA expression by promoter hypermethylation and its association with biologically aggressive phenotype in human breast cancers. Clin Cancer Res. 2005 Feb;11(4):1380-5.

73 Lehn S, Tobin NP, Sims AH, Stål O, Jirström $\mathrm{K}$, Axelson $\mathrm{H}$, et al. Decreased expression of Yes-associated protein is associated with outcome in the luminal A breast cancer subgroup and with an impaired tamoxifen response. BMC Cancer. 2014 Feb;14(1):119.

74 Chang SS, Yamaguchi H, Xia W, Lim SO, Khotskaya Y, Wu Y, et al. Aurora A kinase activates YAP signaling in triple-negative breast cancer. Oncogene. 2017 Mar;36(9): 1265-75.

75 Chang C, Goel HL, Gao H, Pursell B, Shultz $\mathrm{LD}$, Greiner DL, et al. A laminin 511 matrix is regulated by TAZ and functions as the ligand for the $a 6 \mathrm{~B} \beta 1$ integrin to sustain breast cancer stem cells. Genes Dev. 2015 Jan;29(1):1-6.

76 Maugeri-Saccà M, De Maria R. Hippo pathway and breast cancer stem cells. Crit Rev Oncol Hematol. 2016 Mar;99:115-22. 
77 Bartucci M, Dattilo R, Moriconi C, Pagliuca A, Mottolese M, Federici G, et al. TAZ is required for metastatic activity and chemoresistance of breast cancer stem cells. Oncogene. 2015 Feb;34(6):681-90.

78 Taccioli C, Sorrentino G, Zannini A, Caroli J, Beneventano D, Anderlucci L, et al. MDP, a database linking drug response data to genomic information, identifies dasatinib and statins as a combinatorial strategy to inhibit YAP/TAZ in cancer cells. Oncotarget. 2015 Nov;6(36):38854-65.

79 Li YW, Xu J, Zhu GY, Huang ZJ, Lu Y, Li XQ et al. Apigenin suppresses the stem cell-like properties of triple-negative breast cancer cells by inhibiting YAP/TAZ activity. Cell Death Discov. 2018 Nov;4(1):105

80 Ruela-de-Sousa RR, Fuhler GM, Blom N, Ferreira $\mathrm{CV}$, Aoyama $\mathrm{H}$, Peppelenbosch MP. Cytotoxicity of apigenin on leukemia cell lines: implications for prevention and therapy. Cell Death Dis. 2010;1(1):e19.

81 Overholtzer M, Zhang J, Smolen GA, Muir B, Li W, Sgroi DC, et al. Transforming properties of YAP, a candidate oncogene on the chromosome 11q22 amplicon. Proc Natl Acad Sci USA. 2006 Aug;103(33):12405-10.
82 Wang H, Du YC, Zhou XJ, Liu H, Tang SC. The dual functions of YAP-1 to promote and inhibit cell growth in human malignancy. Cancer Metastasis Rev. 2014 Mar;33(1):17381.

83 Yuan M, Tomlinson V, Lara R, Holliday D, Chelala C, Harada T, et al. Yes-associated protein (YAP) functions as a tumor suppressor in breast. Cell Death Differ. 2008 Nov;15(11): 1752-9.

84 Yu SJ, Hu JY, Kuang XY, Luo JM, Hou YF, Di $\mathrm{GH}$, et al. MicroRNA-200a promotes anoikis resistance and metastasis by targeting YAP1 in human breast cancer. Clin Cancer Res. 2013 Mar;19(6):1389-99.

85 Kim T, Lim DS. The SRF-YAP-IL6 axis promotes breast cancer stemness. Cell Cycle. 2016 May;15(10):1311-2.

86 Vlahov N, Scrace S, Soto MS, Grawenda AM, Bradley L, Pankova D, et al. Alternate RASSF1 Transcripts Control SRC Activity, E-Cadherin Contacts, and YAP-Mediated Invasion. Curr Biol. 2015 Dec;25(23):3019-34.
87 Pefani DE, Pankova D, Abraham AG, Grawenda AM, Vlahov N, Scrace S, et al. TGF- $\beta$ Targets the Hippo Pathway Scaffold RASSF1A to Facilitate YAP/SMAD2 Nuclear Translocation. Mol Cell. 2016 Jul;63(1):15666.

88 Lin Q, Yang W. The Hippo-YAP/TAZ pathway mediates geranylgeranylation signaling in breast cancer progression. Mol Cell Oncol. 2016 Mar;3(3):e969638.

89 Mi W, Lin Q, Childress C, Sudol M, Robishaw J, Berlot $\mathrm{CH}$, et al. Geranylgeranylation signals to the Hippo pathway for breast cancer cell proliferation and migration. Oncogene. 2015 Jun;34(24):3095-106.

90 Gartel AL. A new target for proteasome inhibitors: FoxM1. Expert Opin Investig Drugs. 2010 Feb;19(2):235-42.

91 Pandit B, Gartel AL. FoxM1 knockdown sensitizes human cancer cells to proteasome inhibitor-induced apoptosis but not to autophagy. Cell Cycle. 2011 Oct;10(19):3269-73.

92 Tian J, Raffa FA, Dai M, Moamer A, Khadang B, Hachim IY, et al. Dasatinib sensitises triple negative breast cancer cells to chemotherapy by targeting breast cancer stem cells. Br J Cancer. 2018 Dec;119(12):1495-507. 